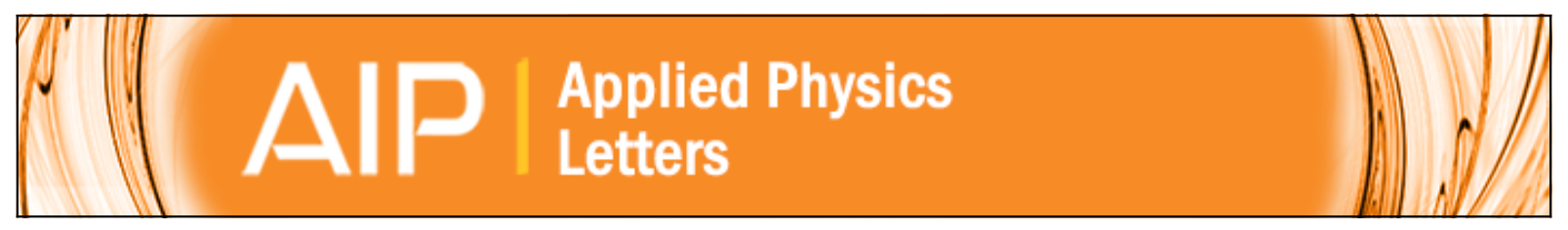

\title{
Maximum power of quantum dot laser versus internal loss
}

Levon V. Asryan

Citation: Applied Physics Letters 88, 073107 (2006); doi: 10.1063/1.2174103

View online: http://dx.doi.org/10.1063/1.2174103

View Table of Contents: http://scitation.aip.org/content/aip/journal/apl/88/7?ver=pdfcov

Published by the AIP Publishing

\section{Articles you may be interested in}

Effect of internal optical loss on the modulation bandwidth of a quantum dot laser

Appl. Phys. Lett. 100, 131106 (2012); 10.1063/1.3697683

Two-state switching and dynamics in quantum dot two-section lasers

J. Appl. Phys. 100, 113104 (2006); 10.1063/1.2397293

Comparison between multilayered InAs quantum dot lasers with different dot densities

Appl. Phys. Lett. 88, 241117 (2006); 10.1063/1.2213520

Limitations on standard procedure of determining internal loss and efficiency in quantum dot lasers

J. Appl. Phys. 99, 013102 (2006); 10.1063/1.2159072

Optical loss and lasing characteristics of high-quality-factor AIGaAs microdisk resonators with embedded quantum dots

Appl. Phys. Lett. 86, 151106 (2005); 10.1063/1.1901810

\section{AlP Re-register for Table of Content Alerts}

\section{Create a profile. \\ Sign up today!}

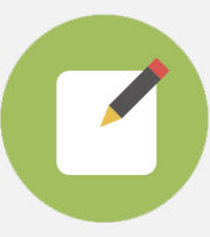




\title{
Maximum power of quantum dot laser versus internal loss
}

\author{
Levon V. Asryan ${ }^{\mathrm{a})}$ \\ Virginia Polytechnic Institute and State University, Blacksburg, Virginia 24061
}

(Received 10 August 2005; accepted 3 January 2006; published online 15 February 2006)

\begin{abstract}
Internal loss, which increases with the carrier density outside the active region, causes a rollover of the light-current curve and strongly limits the output power of a diode laser with a single layer of quantum dots. The maximum power is calculated as a steeply decreasing function of internal loss cross section. The use of multiple layers of quantum dots is shown to significantly improve the laser output characteristics. (C) 2006 American Institute of Physics. [DOI: 10.1063/1.2174103]
\end{abstract}

Internal optical loss is among the key factors controlling the performance of injection lasers. ${ }^{1,2}$ In view of the variety and relative strength of contributing processes, the internal loss coefficient $\alpha_{\text {int }}$ (referred to simply as the internal loss) can range from $1 \mathrm{~cm}^{-1}$ (Ref. 3) to above $20 \mathrm{~cm}^{-1}$. Even for similar structures, there is a wide scatter in reported data for $\alpha_{\text {int }}$. Due to the variety of loss mechanisms, the dependence of $\alpha_{\text {int }}$ on laser-structure parameters is also hard to obtain, which is to say that $\alpha_{\text {int }}$ is not easily adjustable in a given structure. This motivates the study of the extent to which the output power can be affected by the internal loss. Here, the effective cross section $\sigma_{\text {int }}$ for internal absorption loss processes is changed within a typical range ${ }^{5,6}$ and the lightcurrent curves (LCCs) of a quantum dot (QD) laser are calculated for that range of $\sigma_{\text {int }}$. The maximum power is calculated as a function of $\sigma_{\text {int }}$. To the best of my knowledge, no analysis of such a functional dependence was performed even for extensively studied quantum-well lasers. It is shown in this letter that the internal loss, which increases with the carrier density outside the active region, can cause a rollover of the LCC and strongly limits the output power of a QD laser.

The internal loss is presented as ${ }^{7,8}$

$$
\alpha_{\text {int }}=\alpha_{0}+\sigma_{\text {int }} n,
$$

where $\alpha_{0}$ is the constant component, which can be caused by scattering at rough surfaces or absorption in the cladding layers, and $n$ is the free-carrier density in the optical confinement layer (OCL). The component $\sigma_{\text {int }} n$, which increases linearly with $n$, can be caused by free-carrier and intervalence band absorption in the OCL. In general, separate terms for electrons and holes should enter into the right-hand side of Eq. (1). For simplicity, Eq. (1) is used here assuming that $\sigma_{\text {int }} n$ refers to the carrier type dominant in absorption.

The output optical power of a diode laser is

$$
P=\hbar \omega \frac{c}{\sqrt{\epsilon_{g}}} \beta N,
$$

where $\hbar \omega$ is the photon energy, $c$ is the light velocity in a vacuum, $\sqrt{\epsilon_{g}}$ is the group index of the dispersive OCL material, $\beta$ is the mirror loss, and $N$ is the number of photons in the lasing mode.

To calculate the number of photons $N$, the following set of rate equations is used for carriers confined in QDs, free carriers in the OCL, and photons:

$$
\begin{aligned}
\frac{\partial f_{n}}{\partial t}= & \sigma_{n} v_{n} n\left(1-f_{n}\right)-\sigma_{n} v_{n} n_{1} f_{n}-\frac{f_{n}^{2}}{\tau_{\mathrm{QD}}}-\frac{c}{\sqrt{\epsilon_{g}}} \frac{g^{\max }}{N_{S} S}\left(2 f_{n}\right. \\
& -1) N \\
\frac{\partial n}{\partial t}= & \sigma_{n} v_{n} n_{1} \frac{N_{S}}{b} f_{n}-\sigma_{n} v_{n} n \frac{N_{S}}{b}\left(1-f_{n}\right)-B n^{2}+\frac{j}{e b}, \\
\frac{\partial N}{\partial t}= & \frac{c}{\sqrt{\epsilon_{g}}} g^{\max }\left(2 f_{n}-1\right) N-\frac{c}{\sqrt{\epsilon_{g}}}\left(\beta+\alpha_{\text {int }}\right) N
\end{aligned}
$$

where $\sigma_{n}$ is the cross section of carrier capture into a QD, $v_{n}$ is the carrier thermal velocity, $f_{n}$ is the confined-carrier level occupancy in a $\mathrm{QD}, \tau_{\mathrm{QD}}$ is the spontaneous radiative lifetime in a QD, $n_{1}=N_{c} \exp \left(-E_{n} / T\right), N_{c}$ is the effective density of states in the OCL, $E_{n}$ is the carrier excitation energy from a QD, the temperature $T$ is measured in units of energy, $N_{S}$ is the surface density of QDs, $S=W L$ is the cross section of the junction (the QD layer area), $W$ is the lateral size of the device (the QD layer width), $L$ is the cavity length (the QD layer length), $g^{\max }$ is the maximum (saturation) value of the modal gain, $b$ is the OCL thickness, $B$ is the radiative constant for the OCL, and $j$ is the injection current density.

$\mathrm{GaInAsP} / \mathrm{InP}$ heterostructure lasing near $1.55 \mu \mathrm{m}$ is considered here for illustration. ${ }^{7-10}$ The materials of claddings, OCL, and QDs are InP, $\mathrm{Ga}_{0.21} \operatorname{In}_{0.79} \mathrm{As}_{0.46} \mathrm{P}_{0.54}$, and $\mathrm{Ga}_{0.47} \mathrm{In}_{0.53} \mathrm{As}$, respectively. A Gaussian QD-size distribution is assumed. Typical values are taken for the mean linear size of QDs (150 A) and the variance (10\% QD-size fluctuations). Room-temperature continuous-wave operation is assumed. The waveguide layer (OCL) thickness and the surface density of QDs are $b=0.28 \mu \mathrm{m}$ and $N_{S}=6.11$ $\times 10^{10} \mathrm{~cm}^{-2}$, respectively. In the absence of carrier-densitydependent internal loss, these values of $b$ and $N_{S}$ correspond to the optimized design of the laser with a single layer of QDs minimizing the threshold current density. ${ }^{10}$ The optimum parameters and the minimum threshold current density will be affected by the carrier density-dependent internal loss. The optimization in the presence of such loss, although being an important issue, is out of scope of this short letter. At the lasing wavelength $(\lambda=1.58 \mu \mathrm{m})$, the real part of the dielectric constant in the cladding and waveguide layers is 10 and 11.112, respectively. At the above parameters, the optical confinement factor in the structure with a single layer of QDs (along the transverse direction in the waveguide) is $\Gamma$ $=0.024$, and the maximum modal gain is $g^{\max }=29.52 \mathrm{~cm}^{-1}$. The constant component of the internal loss is assumed to be 


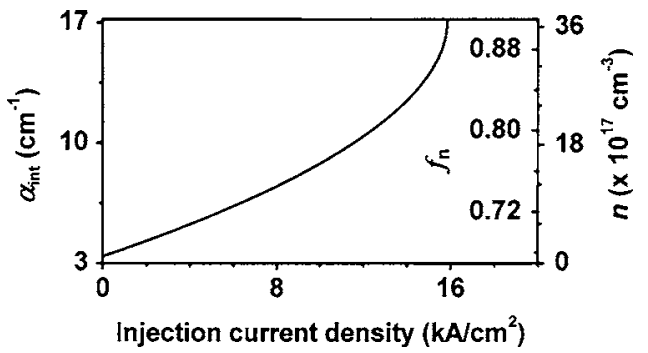

FIG. 1. Confined-carrier level occupancy in a QD (right axis), free-carrier density in the OCL (right axis), and internal loss (left axis) against injection current density. GaInAsP/InP heterostructure with a single layer of QDs emitting near $1.55 \mu \mathrm{m}$ is considered. The parameters are as follows: $10 \%$ QD-size fluctuations, $N_{S}=6.11 \times 10^{10} \mathrm{~cm}^{-2}, \beta=7 \mathrm{~cm}^{-1}$, and $\alpha_{0}=3 \mathrm{~cm}^{-1}$. The normalized cross section of internal absorption loss $\sigma_{\text {int }} / \sigma_{\text {int }}^{\max }=0.1$, where $\sigma_{\text {int }}^{\max }=3.821 \times 10^{-17} \mathrm{~cm}^{2}$ is the maximum tolerable value of the absorption cross section, at which the lasing becomes unattainable in a singlelayer structure considered.

$\alpha_{0}=3 \mathrm{~cm}^{-1}$, which is a typical value; the other parameters are $W=2 \mu \mathrm{m}, L=1.628 \mathrm{~mm}$, and $\beta=7 \mathrm{~cm}^{-1}$. In the structure with four layers of QDs (all other parameters being the same as in the structure with a single layer of QDs), $\Gamma$ is about four times higher, and so is $g^{\max }$.

The confined-carrier level occupancy in a QD, freecarrier density in the OCL, and internal loss, calculated using Eqs. (3) - (5) in a steady state $(\partial / \partial t=0)$, are shown in Fig. 1 against injection current density for a laser with a single layers of QDs. The carrier density and the internal loss increase by more than 30 and 5 times, respectively, with increasing the current above the lasing threshold. This raise in $n$ and $\alpha_{\text {int }}$ dramatically affects the output power (see below). The highest current in the figure is that at which the lasing is quenched.

The output power against injection current density (LCC) is shown in Fig. 2 for lasers with [Fig. 2(a)] single layer of QDs and [Fig. 2(b)] four layers of QDs at different values of the cross section $\sigma_{\text {int }}$ of internal absorption loss

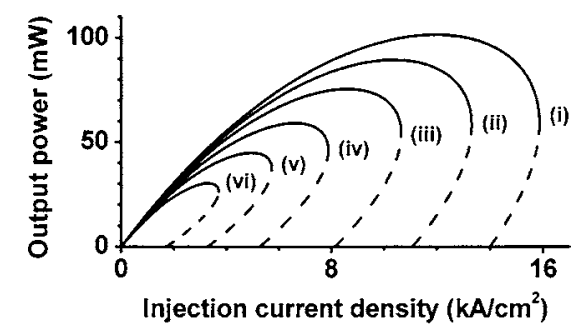

(a)

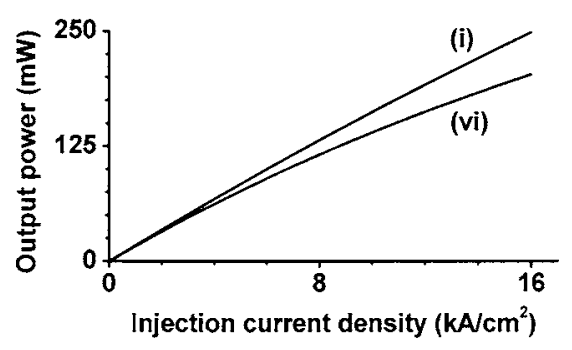

(b)

FIG. 2. Light-current characteristics of lasers with (a) single layer of QDs and (b) four layers of QDs for different cross sections of internal absorption loss. The normalized cross section $\sigma_{\text {int }} / \sigma_{\text {int }}^{\max }$ values are as follows: (i) 0.1 , (ii) 0.112 , (iii) 0.13 , (iv) 0.16, , (v) 0.2 and (vi) $0.27, \sigma_{\mathrm{ino}}^{\max }=3.821$ $\times 10^{-17} \mathrm{~cm}^{2}$.

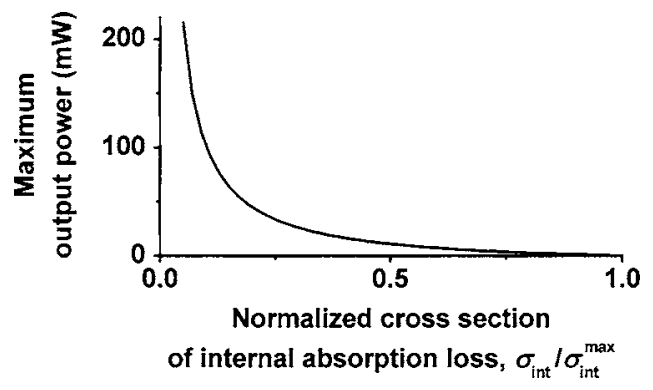

FIG. 3. Maximum output power of a single-layer structure against normalized cross section $\sigma_{\text {int }} / \sigma_{\text {int }}^{\max }$ of internal absorption loss. $\sigma_{\text {int }}^{\max }=3.821$ $\times 10^{-17} \mathrm{~cm}^{2}$.

(presented in the figure caption). The following conclusions can be made for a laser with a single layer of QDs [Figs. 2(a) and 3].

(1) The carrier-density-dependent internal loss in the OCL adds to the intrinsic sublinearity of the LCC. This sublinearity is inherent in heterostructure lasers with reduced-dimensionality active region; ${ }^{9}$ it stems from the absence of pinning of the carrier density $n$ outside the active region above the lasing threshold (see, e.g., Refs. 11-14 for experimental observation of this in quantumwell lasers) and from the superlinearity of the recombination rate with respect to $n$ (this rate is quadratic in $n$ for spontaneous radiative recombination and cubic for nonradiative Auger recombination). In the presence of internal loss, the raise in the carrier density above the lasing threshold (Fig. 1) and hence the sublinearity mechanism are more strongly pronounced.

(2) Further still, the carrier-density-dependent internal loss causes a rollover of the LCC at high injection currents [Fig. 2(a)]. With increasing $j$, the output power first increases and approaches its maximum and then goes down. Note that no heating effect is included into the model. The rollover here is purely due to the internal loss, which increases with the carrier density $n$ in the OCL. Inclusion of heating will reduce the pump current values, at which rollover occurs. The upper (conventional) branch of the LCC [solid portion of curves in Fig. $2(a)$ is discussed here since the maximum power is studied. As shown in Ref. 7, in any structure where $n$ does not pin in the presence of light generation, the $n$-dependence of $\alpha_{\text {int }}$ gives rise to the existence of a second lasing threshold above the conventional threshold. Above the second threshold, the LCC is two valued [solid and dashed portions of curves in Fig. 2(a)].

(3) As seen in Fig. 2(a), the maximum value of the dependence of $P$ on $j$ decreases with increasing internal loss. This is shown in Fig. 3 in an explicit form, where the maximum power is plotted against cross section of internal absorption loss. As $\sigma_{\text {int }}$ approaches a certain value (denoted by $\sigma_{\text {int }}^{\max }$ ), the maximum power reduces to zero, i.e., the lasing becomes unattainable. This quantity $\sigma_{\text {int }}^{\max }$ is the maximum tolerable value of $\sigma_{\text {int }}$; for $\sigma_{\text {int }}>\sigma_{\text {int }}^{\max }$, no lasing is attainable in the structure (in Ref. 8, $\sigma_{\text {int }}^{\max }$ was discussed in the context of the threshold current dependence on $\sigma_{\text {int }}$ ). 
In Fig. 2(b), the LCC of a laser with four layers of QDs is shown for the same range of injection currents as in Fig. 2(a) and for two (highest and lowest) values of $\sigma_{\text {int }}$ used in Fig. 2(a). Here, in contrast to a single-layer structure [ Fig. $2(\mathrm{a})]$, there is no significant difference between the curves calculated for different values of $\sigma_{\text {int }}$. There is neither rollover nor the second branch of the LCC at the injection currents of interest. This is because the above phenomena are controlled by the competition between the maximum modal gain $g^{\max }$ and the internal loss. The maximum modal gain is approximately proportional to the number of layers with QDs. Hence, the larger the number of QD layers (i.e., the higher $g^{\max }$ ) or the lower $\sigma_{\text {int }}$, the higher the currents, at which rollover occurs and the second branch appears. It is apparent from Figs. 2(a) and 2(b) that considerably improved power characteristics (more linear LCC, higher slope efficiency, and maximum power) are obtained in a structure with multiple layers of QDs.

To neatly separate the effect of $\alpha_{\text {int }}$ on the optical power, the model of three rate equations [Eqs. (3)-(5)] is used in this letter, which does not account for the fact that the electron and hole level occupancies can be different in QDs and so can be their densities in the OCL. The inclusion of occupancies and densities separately for electrons and holes will considerably complicate the analysis; at the same time, the main deductions of the present work will remain qualitatively valid.

In conclusion, the carrier-density-dependent internal loss outside QDs has been shown to cause a rollover of the LCC and strongly limit the output power of a laser with a single layer of QDs. The maximum power reduces to zero as the cross section of internal loss approaches its maximum tolerable value. The use of multiple layers of QDs has been shown to significantly improve the laser output characteristics. Internal loss in the bulk OCL has been discussed here. The same conclusions hold for internal loss, which increases with the carrier density in the two-dimensional wetting layer.

This work was supported by the U.S. Army Research Office under Grant No. W911-NF-05-1-0308.

${ }^{1}$ G. P. Agrawal and N. K. Dutta, Long-Wavelength Semiconductor Lasers (Van Nostrand Reinhold, New York, 1986).

${ }^{2}$ L. A. Coldren and S. W. Corzine, Diode Lasers and Photonic Integrated Circuits (Wiley, New York, 1995).

${ }^{3}$ J. J. Lee, L. J. Mawst, and D. Botez, J. Cryst. Growth 249, 100 (2003).

${ }^{4}$ G. E. Shtengel and D. A. Ackerman, Electron. Lett. 31, 1157 (1995).

${ }^{5}$ M. Asada, A. Kameyama, and Y. Suematsu, IEEE J. Quantum Electron. 20, 745 (1984).

${ }^{6}$ D. A. Ackerman, G. E. Shtengel, M. S. Hybertsen, P. A. Morton, R. F. Kazarinov, T. Tanbun-Ek, and R. A. Logan, IEEE J. Sel. Top. Quantum Electron. 1, 250 (1995)

${ }^{7}$ L. V. Asryan and S. Luryi, Appl. Phys. Lett. 83, 5368 (2003).

${ }^{8}$ L. V. Asryan and S. Luryi, IEEE J. Quantum Electron. 40, 833 (2004).

${ }^{9}$ L. V. Asryan, S. Luryi, and R. A. Suris, Appl. Phys. Lett. 81, 2154 (2002).

${ }^{10}$ L. V. Asryan and R. A. Suris, Semicond. Sci. Technol. 11, 554 (1996).

${ }^{11}$ D. Z. Garbuzov, A. V. Ovchinnikov, N. A. Pikhtin, Z. N. Sokolova, I. S. Tarasov, and V. B. Khalfin, Sov. Phys. Semicond. 25, 560 (1991).

${ }^{12}$ W. Rideout, W. F. Sharfin, E. S. Koteles, M. O. Vassell, and B. Elman, IEEE Photonics Technol. Lett. 3, 784 (1991).

${ }^{13}$ N. Tessler, R. Nagar, G. Eisenstein, S. Chandrasekhar, C. H. Joyner, A. G. Dentai, U. Koren, and G. Raybon, Appl. Phys. Lett. 61, 2383 (1992).

${ }^{14}$ H. Hirayama, J. Yoshida, Y. Miyake, and M. Asada, Appl. Phys. Lett. 61, 2398 (1992). 